\title{
Collision Dynamics of Two Barchan Dunes Simulated Using a Simple Model
}

\author{
Atsunari KATSUki $^{12 *}$, Hiraku Nishimori ${ }^{3}$, Noritaka ENDO ${ }^{4}$ and Keisuke TANiguChi ${ }^{4}$ \\ 1 Department of Physics, Osaka University, Osaka 560-0043 \\ ${ }^{2}$ Cybermedia Center, Osaka University, Osaka 560-0043 \\ 3 Department of Mathematical Sciences, Osaka Prefecture University, Sakai 599-8531 \\ ${ }^{4}$ Department of Earth and Space Sciences, Osaka University, Osaka 560-0043
}

\begin{abstract}
The collision processes of two crescentic dunes called barchans are systematically studied using a simple computer simulation model. The simulated processes, coalescence, ejection and reorganization, qualitatively correspond to those observed in a water tank experiment. Moreover we found the realized types of collision depend both on the mass ratio and on the lateral distance between barchans under initial conditions. A simple set of differential equations to describe the collision of one-dimensional (1D) dunes is introduced.
\end{abstract}

KEYWORDS: sand dune, barchan dune, collision process, water tank experiment, saltation, avalanche, numerical simulation

Many types of sand dunes are found in deserts, on the sea bottom and even on Mars. Dunes are formed by interactions between the flow of wind or water and sand. ${ }^{1}$ The flow makes the shape of a dune by transporting sand particles. The wind transports sand particles and forms the shape of dunes. The dune topography, in turn, acts as a boundary condition on the air flow. One of the most intensively studied types of sand dune is the crescentic dune called the barchan. A steadily blowing unidirectional wind generates barchans when the amount of available sand is insufficient for covering the entire bedrock. ${ }^{2}$ Barchans usually migrate as a group, interacting with one another through collisions and interdune sand flow. ${ }^{1,3-11}$ Previous studies, however, have focused mainly on single barchans. ${ }^{12-18}$ One of the reasons is that, because of the slow time scale of the system, it is hard to observe the whole process of the interaction dynamics of barchans in a desert. Recently, some underwater experiments have succeeded in creating barchans, ${ }^{19-22}$ which has enabled real-time observation. In this letter, taking only dominant factors into account, we propose an effective model to simulate the coaxial and offset collisions of two barchans, which are observed in the water tank experiments. ${ }^{22}$

In the model, the dune field is divided into square cells. ${ }^{3-5}$ Each cell is considered to represent an area of the ground which is sufficiently larger than the sand grains. A field variable $h(x, y, t)$ which expresses the local surface height is assigned to each cell; $t$ denotes the discrete time step and the spatial coordinates $x$ and $y$ denote the positions of the center of a cell in the flow and the lateral directions, respectively. The edge length of the cell is taken as a unit of length. In short, $x, y$ and $t$ are discrete variables while $h(x, y, t)$ takes a continuous value. This model belongs to a class of simulation models called cell dynamics. ${ }^{23}$ The motion of sand grains is realized by two processes: saltation and avalanche. Saltation is the transportation process of sand grains by flow. The saltation length and saltation mass are denoted $L$ and

*E-mail address:katsuki@cp.cmc.osaka-u.ac.jp
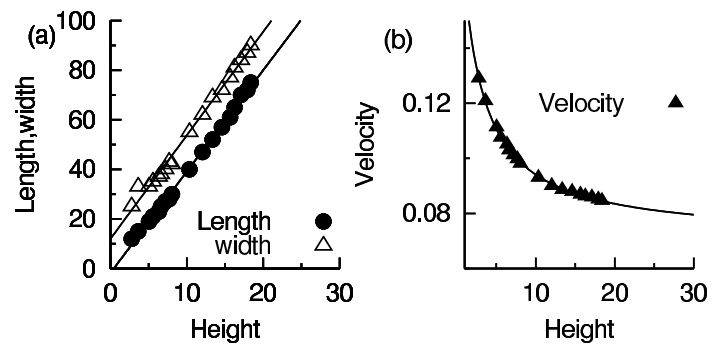

Fig. 1. (a) Relationships between height, length and width of barchans. The solid lines are the best linear fit. (b) Relationships between barchan velocity $(\mathrm{V})$ and barchan height $(\mathrm{H})$. The solid line is the best fit using the relation $V=Q /\left(H+H_{c}\right)+V_{c}$, where $H_{c}$ and $V_{c}$ are phenomenological parameters. ${ }^{24}$

$q$, respectively. Saltation occurs only for cells on the upwind face of dunes. In each time step of the simulation, the amount of sand $q$ is shifted from a cell $(x, y)$ to the leeward cell $(x+L, y)$, which is the numerical expression of a saltation process. Hence, the changes in height, $h(x, y) \rightarrow h(x, y)-q$ and $h(x+L, y) \rightarrow h(x+L, y)+q$, take place at the taking-off cell and the landing cell, respectively. The saltation length $L$ and the amount of transported sand $q$ are modeled by the following rules,

$$
\begin{aligned}
L & =a+b h(x, y, t)-c h^{2}(x, y, t), \\
q & =d,
\end{aligned}
$$

where $\mathrm{a}=1.0, \mathrm{~b}=1.0, \mathrm{c}=0.01$ and $\mathrm{d}=0.1$ are phenomenological parameters. In eq. (1), the second term shows that sand is transported farther away as the height of the sand surface is higher. The last term is introduced for $L$ not to become too large. Note that (1) is used only in the range where $L$ increases as a function of $h(x, y, t)$. The saltation mass $q$ is fixed at 0.1 for simplicity. In the avalanche process, on the other hand, the sand grains slide down along the locally steepest slope until the slope relaxes to be (or be lower than) the angle of repose which is set to be $34^{\circ}$.

We examine if some basic features of barchan are realized by this simple model. ${ }^{12,14,15}$ Starting from an initial 
(a)

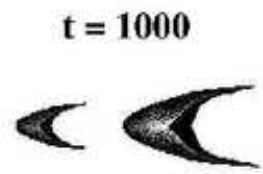

(b)

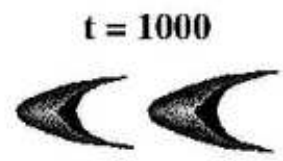

(c)

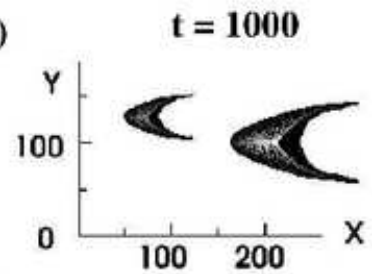

(d)

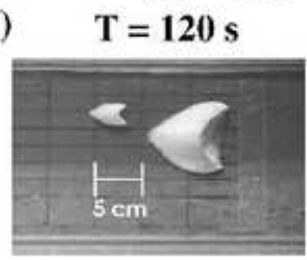

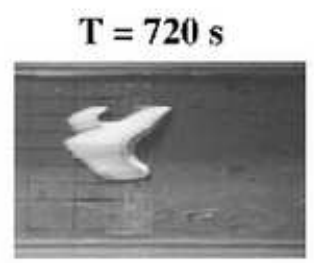
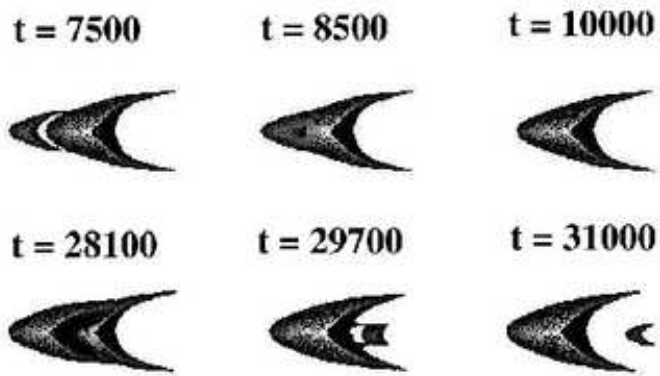

$t=29700$

$\mathbf{t}=\mathbf{3 1 0 0 0}$
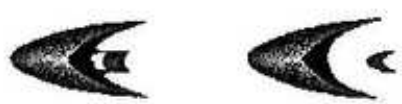

$t=9500$

$t=10700$

$t=12500$
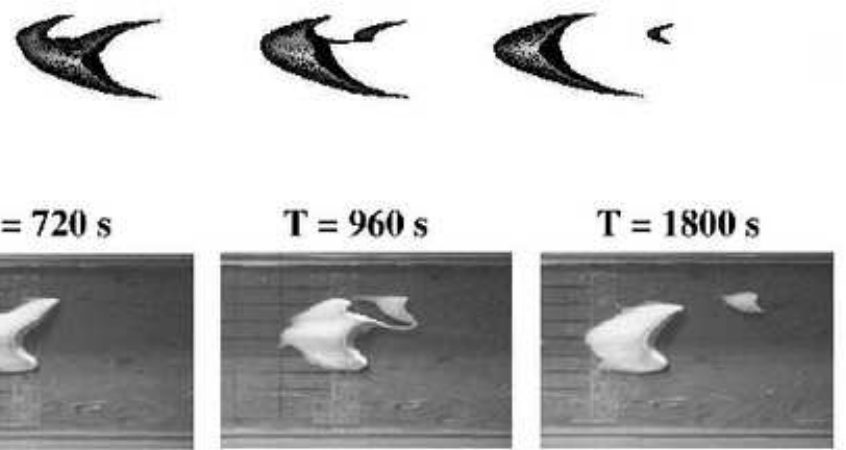

Fig. 2. Typical collisions of two barchans: (a) coalescence process simulated with offset parameter $\alpha=0.0$ and mass ratio $\beta=0.2$, (b) ejection process simulated with offset parameter $\alpha=0.0$ and mass ratio $\beta=0.7$, (c) reorganization process simulated $\alpha=0.3$ and $\beta=0.2$, (d) offset collision performed in water tank which is $10 \mathrm{~m}$ long, $20 \mathrm{~cm}$ wide and $50 \mathrm{~cm}$ deep with water depth maintained at approximately $13 \mathrm{~cm} \cdot{ }^{22}$ In (a), (b) and (c) the slopes at the angle of repose are painted in black.

Gaussian sand pile, we measured the morphologic relations after a steady barchan shape was reached. The linear relationships between height, length (Fig. 1(a)) and width(Fig. 1(b)) are shown. Next, the roughly inverse relationship between migration velocity and height(Fig. 1(c)) was confirmed to hold. In these tests, quasi-periodic boundary conditions were used in which the total mass of sand flowing away from the downwind and lateral boundaries was homogeneously re-injected from the upwind boundary.

In order to simulate collision processes, two initial barchans are situated in a field. The longitudinal (i.e, wind directional) distance between their crests is set to $d_{x}=2 L_{l}$, where $L_{l}$ is the main body length of the initial leeward barchan, while the lateral distance between them is set to $d_{y}=\alpha W_{l}$, where $W_{l}$ is the width of the same leeward barchan. The constant $\alpha$ is varied as a control parameter which we call the offset parameter. We fix the initial mass of the leeward barchan $\left(M_{l}=10\right)$ and define its ratio $\left(\beta \equiv M_{w} / M_{l}\right)$ to the initial mass of the windward barchan $\left(M_{w}\right)$ as another control parameter.

In the first simulation, we examine the coaxial collisions. For the sake of quantitative comparison with experiments, open boundary conditions are used. This means that there is no influx sand from the upwind boundary and the sand out of the numerical field from the downwind boundary is neglected. Figure 2(a) shows the simulated process for $\alpha=0.0$ and $\beta=0.2$. After two barchans collide with each other, the windward barchan
Table I. Calculated results classified into coalescence $(x)$, ejection $(\triangle)$ and reorganization $(\circ)$ for various mass ratios and offset parameters.

\begin{tabular}{cccccccc} 
Offset & \multicolumn{7}{c}{ Mass $\operatorname{ratio}(\beta)$} \\
parameter $(\alpha)$ & 0.1 & 0.2 & 0.3 & 0.4 & 0.5 & 0.6 & 0.7 \\
\hline 0 & $\times$ & $\times$ & $\times$ & $\times$ & $\times$ & $\triangle$ & $\triangle$ \\
0.1 & $\times$ & $\times$ & $\circ$ & $\circ$ & $\circ$ & $\circ$ & $\circ$ \\
0.2 & $\times$ & $\circ$ & $\circ$ & $\circ$ & $\circ$ & $\circ$ & $\circ$ \\
0.3 & $\circ$ & $\circ$ & $\circ$ & $\circ$ & $\circ$ & $\circ$ & $\circ$ \\
0.4 & $\circ$ & $\circ$ & $\circ$ & $\circ$ & $\circ$ & $\circ$ & $\circ$ \\
0.5 & $\circ$ & $\circ$ & $\circ$ & $\circ$ & $\circ$ & $\circ$ & $\circ$
\end{tabular}

is absorbed into the leeward barchan, during which the upwind slope of the leeward barchan is temporally eroded and forms a dent. As time proceeds, the dent is filled up and the unified dune recovers the crescent shape of the barchan. The behavior of barchans which is similar to this simulation result was observed in water tank experiments. ${ }^{22}$ Hereafter, we call such a process the coalescence of two barchans. Figure 2(b) shows the time evolution for $\alpha=0.0$ and $\beta=0.7$. Similar to the case of coalescence, in the initial stage, a part of the windward barchan merges into the leeward barchan. However, the valley between two crests is kept imperfectly filled, thereafter, a small barchan is bled from the downwind slope. Although the small barchan firstly drags ridges from its horns connecting to the windward barchan, the ridges are soon cut and finally steady shapes of windward and leeward barchans are attained. Hereafter, we call such a process 

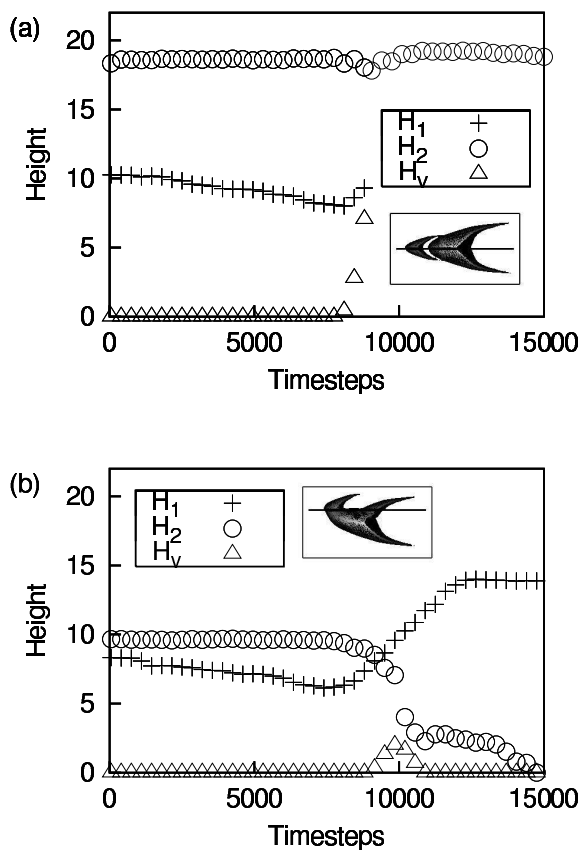

Fig. 3. Time evolution of heights $\boldsymbol{H} \equiv\left(H_{1}, H_{2}, H_{v}\right)$ in longitudinal profile along solid line as indicated in inset. (a) For coaxial collision (Fig. 2(a)) the coalescence of two crests is seen at approximately 9000 time steps. (b) For offset collision (Fig. 2(c)) the exchange of the heights of two crests is seen at approximately 9200 time steps, which indicates the reorganization of two barchans.

the ejection of a small barchan. Figure 2(c) shows the time evolution for $\alpha=0.3$ and $\beta=0.2$. As the initial windward barchan approaches the leeward barchan, the wing of the leeward barchan is partially eroded and is pushed downward. After that it is separated from the deforming main body. The separated body drags a ridge extending from the main body for a while. Subsequently, it is completely isolated and recovers the crescent shape. Hereafter we call such a process the reorganization of two barchans. This process is qualitatively similar to what happened in the experimental tank (Fig. 2(d)). Note that the types of realized collision depend on the mass ratios of initial barchans and on their relative positions.

In addition, systematic calculations are performed with various combinations of $\alpha$ and $\beta$. The obtained collisions are classified into three types, coalescence, ejection and reorganization (Table I). Note that the coalescence appears in the region of low values of offset parameter and small mass ratios, and the reorganization occurs at high values of offset parameter or large mass ratios. On the other hand, ejection occurs at low values of offset parameter and large mass ratios, which corresponds to the "solitary-wave behavior" obtained by the previous simulation. ${ }^{9}$ This diagram should be compared with those of further subaqueous experiments and also to those of field observations.

To explore the intrinsic mechanism underlying the difference between the three types of collision, we focus on the dynamics of longitudinal profiles of barchans particularly on the heights of the windward crest $\left(H_{1}\right)$, the leeward crest $\left(H_{2}\right)$ and the bottom of the valley $\left(H_{v}\right)$. Fig-

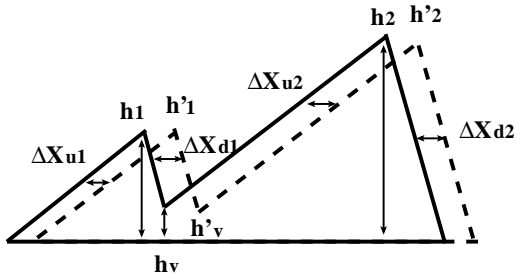

Fig. 4. Schematic diagram for collision process of $1 \mathrm{D}$ barchan collision. The solid and dashed lines represent the profiles at time $t$ and time $t+\Delta t$, respectively.

ure 3(a) shows the time evolution of $\boldsymbol{H} \equiv\left(H_{1}, H_{2}, H_{v}\right)$ in the case of coalescence. $H_{v}$ reaches $H_{1}$ at approximately 9000 time steps. Figure 3(b) shows the time evolution of $\boldsymbol{H}$ in the case of reorganization. The exchange of the heights of two barchans is seen at approximately 9200 time steps, whereas the bottom height of the valley is kept below them throughout the process. The time evolution in the case of ejection is qualitatively the same as that in the case of reorganization. In short, ejection is a type of reorganization. We discuss only coalescence and reorganization hereafter.

The dynamics in each profile of Fig. 3 is not selfcomplete because of the lateral sand flow. Nevertheless, the dynamics realized in each profile is roughly inferred from the initial condition, that is, (i) if the height ratio of the windward crest to the leeward crest is comparatively small under the initial condition, coalescence occurs. (ii) if the height ratio is comparatively large, the reorganization of two barchans occurs. In process (i), the smaller barchan on the windward side climbs the upward face of the larger barchan on the leeward side and is absorbed on the latter before its crest reaches the same height as the latter. On the other hand, in process (ii), the windward barchan climbs the larger leeward barchan and its crest becomes higher than that of the leeward barchan without absorption into the latter. Subsequently, the leeward barchan runs away from the windward barchan because of the inverse relationship between velocity and height. From the above discussion, the important factor determining the type of collision is presumed to be the competition between the absorption and height exchange of two barchans.

To attain a clear picture of the complex processes of collisions, we further simplify the model using a set of naive assumptions. The first assumption is the geometric similarity between $1 \mathrm{D}$ dunes of different sizes which means that constant angles of upwind and downwind slopes are maintained irrespective of their size. The second assumption is that the sand flux at the crests of dunes is described by the product of eqs. (1) and (2). The third assumption is the mass conservation of sand crossing over a crest, and that all the sand blown across a crest becomes trapped in the downwind face of the same dune without directly escaping to the leeward. The combination of the above assumptions leads to a simple conservation relationship between the eroded sand on the upwind faces and the accumulated sand on the downwind faces of each dune(Fig. 4). The sand mass conservation 

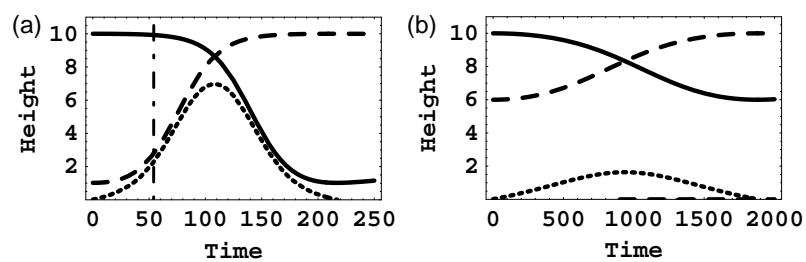

Fig. 5. Time evolution of heights of windward dune(dashed), leeward dune(solid) and valley(dotted) obtained by numerical calculation. The dash-dotted line represents time when $\delta h$ reaches $\delta h_{\text {min }}=0.5$. (a) Under the initial conditions of $h_{1}(0)=1.02$, $h_{2}(0)=10.0$ and $h_{v}(0)=0.01$, coalescence collision occurs. (b) Under the initial conditions of $h_{1}(0)=6.00, h_{2}(0)=10.0$ and $h_{v}(0)=0.01$, the reorganization occurs.

in the windward dune is expressed as,

$\rho \frac{\left(h_{1}+h_{1}^{\prime}\right)}{2} \Delta x_{u 1}=\rho \frac{\left(h_{1}+h_{1}^{\prime}\right)-\left(h_{v}+h_{v}^{\prime}\right)}{2} \Delta x_{d 1} \equiv q_{1} \Delta t$,

where $h_{1}$ and $h_{v}$ are the heights of the windward dune and the bottom of the valley at time $t$, respectively, whereas $h_{1}^{\prime}$ and $h_{v}^{\prime}$ indicate the heights of the windward dune and the bottom of the valley at time $t+\Delta t . \Delta x_{u 1}$ and $\Delta x_{d 1}$ denote the horizontal displacements of the upwind surface and the downwind surface of the windward dune during $\Delta t, q_{1} \equiv q\left(h_{1}\right)$ denotes the sand mass crossing over the crest per unit time, and $\rho$ denotes the area density of the bulk of sand. Also, the change in height of the windward crest for $\Delta t$ is expressed as,

$$
h_{1}^{\prime}-h_{1}=A \Delta x_{d 1}-A \Delta x_{u 1} \equiv \Delta h_{1},
$$

where $A$ is the geometrical constant of similar triangles constituting 1D dunes, namely, the ratio of their heights to the lengths of their bases. Using eqs. (3) and (4) and taking the limits $\Delta t \rightarrow 0, \Delta x_{u 1} \rightarrow 0, \Delta x_{d 1} \rightarrow 0$ and $\Delta h_{1} \rightarrow 0$, the evolution equation of the crest height,

$$
\frac{d h_{1}}{d t}=\frac{q_{1} A}{\rho}\left(\frac{1}{h_{1}-h_{v}}-\frac{1}{h_{1}}\right),
$$

is obtained. Similarly the evolution equations of the heights of the leeward dune and the valley are written as,

$$
\begin{aligned}
\frac{d h_{2}}{d t} & =\frac{q_{2} A}{\rho}\left(\frac{1}{h_{2}}-\frac{1}{h_{2}-h_{v}}\right), \\
\frac{d h_{v}}{d t} & =\frac{q_{1} A}{\rho}\left(\frac{1}{h_{1}-h_{v}}\right)-\frac{q_{2} A}{\rho}\left(\frac{1}{h_{2}-h_{v}}\right) .
\end{aligned}
$$

In addition to the above closed set of differential equations, we set the minimum depth as $\delta h_{\min }$. Here, the valley depth $\delta h$ is defined as $\delta h=h_{1}-h_{v}$ and two dunes are considered to coalesce once $\delta h$ reaches $\delta h_{\min }$. Figure 5 (a) shows a typical collision obtained by the calculation of eqs. (5), (6) and (7) with $\delta h_{\min }=0.5$. In this case, the coalescence of two dunes occurs at the time represented by the dash-dotted line. $h_{v}$ reaches $h_{1}$ before the exchange of heights occurs and coalescence occurs. This process is similar to that indicated in Fig. 3(a). Figure 5 (b) shows another typical collision under the same initial conditions as indicated in Fig. 5(a) except for the higher windward initial dune. The exchange of heights occurs before $h_{v}$ reaches $h_{1}$ and reorganization occurs. This process is similar to that indicated in Fig. 3(b). This indicates that the set of simple differential equations, eqs. (5), (6) and (7), contains the intrinsic features of collision dynamics.

To summarize, the coaxial and offset collisions of two barchans are simulated using a simple model that includes only saltation and avalanche processes without taking complex wind flow into account. Also we introduced a set of differential equations, which effectively describe the collision processes of $1 \mathrm{D}$ dunes.

We thank Macoto Kikuchi for helpful discussions. This work was partially supported by the 21 st Century COE Program, "Towards a new basic science : depth and synthesis" and the Grant-in-Aid for Scientific Research (C) No. 16605008 of the Ministry of Education, Culture, Sports, Science and Technology.

1) R. A. Bagnold : The Physics of Blown Sand and Desert Dunes (Methuen, London, 1941).

2) R. J. Wasson and R. Hyde :Nature 304 (1983) 337

3) H. Nishimori and N. Ouchi :Phys. Rev. Lett. 71 (1993) 197.

4) B. T. Werner :Geology 23 (1995) 1107.

5) H. Nishimori, M. Yamasaki and K. H. Andersen :J. Mod. Phys. B 12 (1998) 256.

6) S. P. Gay: Geomorphology 27 (1999) 279.

7) H. Momiji and R. Carretero-Gonzalez and S. R. Bishop and A. Warren :Earth Surface Processes and Landforms 25 (2000) 905.

8) A. R. Lima, G. Sauermann, H. J. Herrmann and K. Kroy :Physica A 310 (2002) 487.

9) V. Schwämmle and H. J. Herrmann :Nature 426 (2003) 619.

10) J. Shibata and Y-h. Taguchi :J. Phys. Soc. Jpn. 72 (2003) 2685.

11) P. Hersen, K. H. Andersen, H. Elbelrhiti, B. Andreotti, P. Claudin and S. Douady :Phys. Rev. E 69 (2004) 011304.

12) H. J. Finkel: Journal of Geology 67 (1959) 614.

13) F. K. Wippermann and G. Gross :Boundary-Layer Meteorology 36 (1986) 319.

14) P. A. Hesp and K. Hastings :Geomorphology 22 (1998) 193.

15) G. Sauermann, P. Rognon, A. Poliakov and H. J. Herrmann :Geomorphology 36 (2000) 47.

16) B. Andreotti, P. Claudin and S. Douady :Eur. Phys. J. B 28 (2002) 321.

17) K. Kroy, G. Sauermann and H. J. Herrmann :Phys. Rev. E 66 (2002) 031302.

18) G. Sauermann , J. S. Andrade Jr., L. P. Maia, U. M. S. Costa, A. D. Araujo and H. J. Herrmann :Geomorphology 1325 (2003) 1.

19) Y. Niño and M. Barahona :Proc. Int. Assoc. Hydraul. Res. 27B (1997) 1037.

20) P. Hersen, S. Douady and B. Andreotti :Phys. Rev. Lett. 89 (2002) 264301.

21) N. Endo, H. Kubo and T. Sunamura: Earth Surface Processes and Landforms 29 (2004) 31.

22) N. Endo, K. Taniguchi and A. Katsuki :Geophysical Research Letters 31 (2004) 12503.

23) Y. Oono and S. Puri :Phys. Rev. A 38 (1988) 434.

24) H. Momiji and H. Nishimori and S. R. Bishop :Earth Surface Processes and Landforms 27 (2002) 1335. 